2. Mossotto E, Ashton JJ, O'Gorman L, Pengelly RJ, Beattie RM, MacArthur BD, et al. GenePy - a score for estimating gene pathogenicity in individuals using nextgeneration sequencing data. BMC Bioinformatics. 2019 May;20(1):254.

3. Lee $S, W u M C$, Lin $X$. Optimal tests for rare variant effects in sequencing association studies. Biostatistics. 2012 September;13(4);762-775.

\section{P94 TLR7 AND TLR8 DIFFERENTIALLY ACTIVATE THE IRF AND NF-KB PATHWAYS IN SPECIFIC CELL TYPES TO PROMOTE INFLAMMATION}

Andrew T Bender, Evgeni Tzvetkov, Albertina Pereira, Siddha Kasar, Melinda Przetak, Shinji Okitsu, Jaromir Vlach. Immunology, EMD Serono Research and Development Institute, Billerica, USA

\subsection{6/lupus-2020-eurolupus. 138}

Background TLR7 and TLR8 are pattern recognition receptors that reside in the endosome and are activated by ssRNA molecules. TLR7 and TLR8 participate in the anti-viral defense response but their aberrant activation has also been implicated as a driver of autoimmune diseases such as lupus. The receptors have slightly different ligand binding specificities and cellular expression patterns suggesting they have non-redundant specialized roles. How the roles of TLR7 and TLR8 differ in driving disease may be determined by which cell types express the TLRs and how they respond to activation of each.

Methods To delineate the differential effects of TLR7 or TLR8 activation we used gene expression analysis and intracellular cytokine staining to characterize changes induced by TLR specific agonists in different immune cell types. Anti-IFNR antibody treatment was also used in whole blood and lupus mice to further define which responses are a direct consequence of TLR7/8 activation and which are secondary responses driven by Type I interferon or cytokines produced subsequent to the primary response.

Results It was found that the IRF and NF-kB pathways are differentially activated downstream of the TLRs in various cell types. The differential expression and activity of TLR7 vs TLR8 was notable when comparing different DC populations, monocytes, and neutrophils. TLR7 was more biased toward activation of IRF responses while TLR8 activation resulted in stronger NF-kB signaling. The anti-IFNR antibody studies showed that inhibiting IFN activity can block secondary IFN-induced gene expression changes downstream of TLR7/8 activation, but not NF-kB-regulated genes induced directly by TLR7/8 activation at early timepoints.

Conclusions In summary, these results elucidate the ways in which TLR7 and TLR8 regulate immunity and how their activation may shape an immune response. The results also have implications for selecting strategies for treating autoimmune diseases driven by TLR7/8 activation.

\section{P95 COSTIMULATORY MOLECULES ON CMV-SPECIFIC T- CELLS IN CMV IGG+ PATIENTS WITH SYSTEMIC LUPUS ERYTHEMATOSUS}

${ }^{1}$ Antje Savidis, ${ }^{1}$ Benjamin Wilde, ${ }^{1}$ Andreas Kribben, ${ }^{2}$ Oliver Witzke, ${ }^{2}$ Sebastian Dolff. ${ }^{1} D e p t$. of Nephrology, University Hospital Essen, University Duisburg-Essen, Essen; ${ }^{2}$ Dept. of Infectious Diseases, University Hospital Essen, University Duisburg-Essen, Essen, Germany

10.1136/lupus-2020-eurolupus. 139
Background Cytomegalovirus (CMV) infection is an uncommon but severe infection in patients with systemic lupus erythematosus (SLE) due to immunosuppressive therapy. Standard prophylaxis with antiviral agents or pre-emptive strategies to monitor viral load are not standard of care. The aim of the present study was to investigate the expression of coinhibitory molecules PD-1 and BTLA-4 on CMV specific T-cells in SLEpatients.

Methods Twenty-three SLE-patients and eight healthy controls were enrolled. Nineteen SLE patients were CMV IgG+, four were CMV IgG-. Peripheral blood was sampled and stimulated with CMV lysate, SEB or control serum in presence of antiCD28/CD49d. After six hours of stimulation, CD154 expression was determined by flow cytometry on $\mathrm{CD}^{+}{ }^{+} \mathrm{T}$-cells. The coinhibitory molecules PD-1 and BTLA were determined on activated $\mathrm{CD} 154^{+} \mathrm{CD}^{+}$T-cells. Symptomatic CMV infection was defined as CMV syndrome or tissue invasive disease. Asymptomatic CMV infection was defined as detectable CMV replication in peripheral blood and absence of signs indicating CMV syndrome/tissue invasive disease.

Results PD-1 and BTLA-4 expression was not significantly different on $\mathrm{CMV}$-specific $\mathrm{CD} 154^{+} \mathrm{CD} 3^{+} \mathrm{T}$-cells in SLEpatients as compared to healthy controls. An analysis according to the CMV serostatus revealed a tendency to a decreased proportion of $\mathrm{PD}-1^{+} \mathrm{CD} 154^{+} \mathrm{CD} 3^{+}$T-cells in CMV IgG negative patients as compared to CMV IgG positive. The BTLA-4 expression was significantly decreased on $\mathrm{CD} 154^{+} \mathrm{CD} 3{ }^{+}$T-cells in CMV IgG negative patients as compared to CMV IgG positive.

Conclusion SLE-patients show a significant decreased expression of BTLA on CMV-specific T-cells. The co-inhibitors PD-1 and BTLA usually promote T-cell suppression. Thus a decrease may prone to severe symptomatic infections.

\section{P96 THE REGULATION AND PHARMACOLOGICAL MODULATION OF IMMUNE COMPLEX INDUCED PRODUCTION OF TYPE III IFN BY PLASMACYTOID DENDRITIC CELLS}

Karin Hjorton, Niklas Hagberg, Pascal Pucholt, Olof Berggren, Maija-Leena Eloranta, Lars Rönnblom. Dept. of Medical Sciences, Rheumatology, Science for Life Laboratory, Uppsala University, Uppsala, Sweden

\subsection{6/lupus-2020-eurolupus. 140}

Background Acknowledging the importance of type I interferon (IFN) in Systemic Lupus Erythematosus (SLE), we asked if RNA containing immune complexes (RNA-IC), which trigger the IFN- $\alpha$ synthesis by plasmacytoid dendritic cells (pDCs), also activate type III IFN (IFN- $\lambda 1-3$ ) production, and how this is regulated.

Methods Peripheral blood mononuclear cells (PBMCs) were isolated from SLE patients and healthy individuals and depleted of monocytes. Immune cells were isolated from healthy PBMCs. Cells were stimulated with RNA-IC. Cytokines were measured by immunoassays, a microarray of pDCs, NK and B cells, as well as single-cell RNA-sequencing of pDCs was performed.

Results Type III IFN mRNA was induced in RNA-IC stimulated $\mathrm{pDC}-\mathrm{NK}$ and $\mathrm{pDC}-\mathrm{B}$ cell co-cultures, type III IFN was produced in $\mathrm{pDC}$ and $\mathrm{pDC}-\mathrm{NK}$ cell co-culture supernatants. A small subset $(3 \%)$ of RNA-IC activated pDCs expressed both IFNs type III and type I. Priming with IFN- $\lambda 2$, IFN- $\alpha 2 b$, interleukin (IL)-3, IL-6 and granulocyte-macrophage colony 